\title{
Le très curieux silence des Doxographes à propos de l'incompétence des auteurs des opinions chez Parménide
}

\author{
Nestor-Luis Cordero
}

\begin{abstract}
Since the Goddess of Parmenides presents the two ways to explain the reality that must be faced by this who want to become a "man who knows", the truth and the opinions of mortals, she makes clear that the opinions (dóxai) are not "reliable". Later, when he describes in detail how the makers of opinions really are, the description is devastating: they are the people who are incapable of judging, who are astonished, who do not know how to use sensations, and who have a misguided intellect. Consequently, when they express their opinions, they present only a "misleading set of words. However, already from Aristotle onwards, this way of conceiving reality is attributed to Parmenides himself, and not to "the mortals". Theophrastus echoes this interpretation of Aristotle and, with him, the totality of the Doxographers. Obviously, in order to attribute the "opinions" to Parmenides himself, any reference to the incapacity of his authors is absent from the comments: no Doxographer mentions it.
\end{abstract}

Étant donné l'état plus que lacunaire dans lequel nous avons reçu le Poème de Parménide (environ cent soixante et un vers; à peine deux pages d'un texte imprimé !), chaque mot récupérée a un poids considérable, plus important que celui qu'on attend concernant le même mot dans un texte mieux (ou totalement) conservé. Chaque mot est un petit joyau que doit être respecté, même vénéré. Or, ce travail s'occupe de quelques mots que Parménide consacre aux auteurs des dóxai dont le sens péjoratif, d'une manière très curieuse, a disparu des études consacrées à la philosophie de Parménide. Ces quelques mots, clairs et distincts, semblent avoir dérangé les Doxographes. Nous essaierons de trouver la cause de ce curieux silence.

Cela va de soi que notre recherche s'appuie sur une interprétation générale de la pensée de l'Éléate. Notre point de vue sur ce sujet, en deux mots ${ }^{1}$, est le suivant. Comme

\footnotetext{
${ }^{1}$ Nous nous sommes occupés en détail de notre interprétation de Parménide dans grand nombre d'articles et notamment dans Cordero, N. L. (1997², 2004, et 2019).
} 
ses prédécesseurs, Parménide veut expliquer qu'est-ce que la réalité (tà ónta), mais, au lieu de faire appel à des arkhaí, il va en deçà de la recherche de ses collègues et affirme qu'il faut admettre, d'abord, que s'il y a des ónta, c'est parce qu'il y a de l'être (ce que même la langue grecque confirme !). Or, le fait d'être est un état ("être" est un verbe, et un verbe fait allusion à une action ou un état) qui est le fondement de tout ce qui est (car tout ce qui est, est "en train d'être", eón). Et cet état possède une série de caractéristiques que Parménide expose dans une longue citation, heureusement transmise par Simplicius, le fr. 8. Mais le véritable cours de philosophie ${ }^{2}$ qui est son Poème didactique ${ }^{3}$ expose aussi une manière (hodós) ${ }^{4}$ erronée (apatelón, 8. 52) de vouloir expliquer le réel. Il attribue cette habitude aux "autres", des gens (d'autres philosophes? la foule?) qui, par rapport à son porte-parole, qui est une Déesse (donc, immortelle), sont appelés en général "les mortels" (fr. 1.30, fr. 8.39, fr. 8.51, fr. 8.61) et, en deux occasions, "les hommes" (toujours au pluriel: fr. 1.27, fr. 19.3). ${ }^{5}$ Parménide dédie quelques vers à exposer ce point de vue, qui, pour lui, est erroné, et il dit pourquoi il a décidé de consacrer quelques lignes à présenter une "théorie" trompeuse: "je t'exprime tout ce diákosmon semblable afin d'empêcher que n'importe quel point de vue des mortels puisse te dépasser" (fr. 8. 60-1).

Il y a chez Parménide, par conséquent, un "discours (lógos) digne de foi et une pensée autour de la vérité" (8.50-51), qui développe les sémata du fait d'être ([tò] eón), suivi d'une "trompeuse série des mots" (8.52-3) qui expose "les opinions des mortels". Préciser, avec des arguments solides et sans écouter le chant des sirènes des "spécialistes", quels passages récupérés du Poème appartiennent à l'un ou à l'autre des sujets traités (qui ont été groupés en deux "parties" depuis 1795$)^{6}$ va au-delà des étroits limites de ce travail. ${ }^{7}$ Il suffit de dire que nous ne saurons jamais dans quel ordre se trouvait chacun des dix-neuf fragments du Poème récupérés (mis à part le fr. 1), et que l'arrangement

\footnotetext{
${ }^{2}$ Cf. Cordero, N. L. (1990).

3 "Parmenides' choice of the didactic epic as the form best suited to his own philosophy was a bold and extremely significant innovation" (Jaeger, W. 1947, p. 92). Cet aspect a été souligné très récemment par Galgano, N. S. (2020).

${ }^{4}$ Hodós est à la fois "chemin" et "méthode" ou "manière". Le mot anglais "way" a hérité ces deux nuances.

${ }^{5} \mathrm{Au}$ fr. 16 ántropos fait allusion aux "hommes" en général.

${ }^{6}$ Voir Fülleborn, Georg G. (1795).

${ }^{7}$ Nous avons développé notre position in Cordero, N.L. (2013).
} 
devenu aujourd'hui orthodoxe, est totalement inacceptable, car il s'appuie sur une "platonisation" de Parménide de la part de Simplicius. ${ }^{8}$

Étant donné que les idées exposées dans le discours digne de foi, d'une part, et le récit trompeur contenu dans les opinions, d'autre part, sont opposés, ils ne peuvent pas appartenir au même "auteur". Concernant le pistón lógon, la Déesse revendique plusieurs fois son copyright, soit s'exprimant à la première personne ("Il est commun pour moi où je commence", fr. 5; "Je ne te permets pas de dire ni de penser que cela [ $s c$. la naissance de ce qui est] se serait produite à partir de ce qui n'est pas...", fr. 8.8), soit en ordonnant d'accepter son discours: "J'ordonne de proclamer cela [sc., "qu'être est possible et que le néant n'existe pas"], fr. 6.1-2)". Et, concernant les opinions, la Déesse est aussi claire et précise: elle expose les idées d'"autres", afin que, en connaissance de cause, on puisse les éviter. C'est pour cette raison que dans toutes les occasions où la notion de dóxai apparait, elle est accompagnée par une référence à ses auteurs: les "mortels", les "hommes": l'apprenti philosophe doit être informé des "opinions des mortels" (brotôn dóxas, 1.30); "apprends maintenant les opinions des mortels" (dóxas...broteías, 8.51); "ainsi sont nées les choses selon l'opinion (dóxa) [...] Pour chacune les hommes (ánthropoi) ont établi un nom distinctif" (fr. 19).

Il nous semble évident que Parménide, au caractère divin (donc, immortel) de la Déesse, qui exprime "le coeur inébranlable de la vérité"(1.29), veut opposer la fragilité des points de vue (gnómas, 8.53) auxquels sont condamnés les humains. ${ }^{9}$ Leur chemin est éloigné de celui de la Déesse (fr. 1.27), qui a dé-couvert une vérité, peut être "la" vérité ( $c f$. l'étymologie d'alétheia); les "mortels", en revanche, se limitent à placer sur les choses des noms qu'ils "croient" être vrais (pepoithótes eînai alêthê, 8.39). Parménide, de toute évidence, est intéressé à montrer la triste situation dans laquelle se trouvent ces doxophiles car il leur consacre dix vers (6 vers du fr. 6 et 4 vers du fr. 7) à sa description, ce qui est très symptomatique et exceptionnel dans ce qui reste de son Poème.

Regardons la présentation des "créateurs" des dóxai au fr. 6. Nous ne savons pas dans quel endroit du Poème se trouvaient les neuf vers que nous appelons aujourd'hui "fragment 6", mais la nouveauté que Parménide introduit dans l'exposé de ses idées (originalité introuvable avant, soit parce qu'aucun philosophe l'avait mis en place, soit

\footnotetext{
${ }^{8}$ Évidemment nous ne pouvons pas démontrer cette interprétation dans les étroits limites de cet article. Nous renvoyons le lecteur éventuel aux ouvrages cités à la note 1.
} 
étant donné la rareté des texte retrouvés) est le raisonnement, ce qui suggère que quelques citations du Poème auraient dû se trouver nécessairement avant ou après d'autres. Or, après avoir exposé le contenu des seuls chemins qui, a priori (l'un des deux sera par la suite rejeté) peuvent être empruntés par le noûs "pour penser"10, il propose, dans le "fragment 6", de les essayer: il faut commencer par le chemin qui est accompagné par la vérité (ce que la Déesse proposera de faire au fragment 8, jusqu'au vers 50), et il faudra après être au courant aussi de ce que les mortels "croient" (nenómisthai, fr. 6.8), tel que la Déesse l'exposera dans les vers 51 à 59 du fr. 8.

Nous avons dit qu'il faut commencer ${ }^{11}$ par le premier chemin énoncé au fr. 2 (et repris au début du fr. 6), celui qui affirme "qu'il y a de l'être (ésti gàr eînai) et, en revanche, le néant n'existe pas (médén d'ouk esti)" (fr. 6.1b-2a). ${ }^{12}$ Et tout de suite la Déesse dit que le voyage doit re-commencer ${ }^{13}$ ensuite par "[le chemin] façonné par les mortels qui ne savent rien, hommes à deux têtes, car, à l'intérieur de leur poitrine, l'incapacité guide l'intellect (nóos) errant; ils son entraînés aveugles et sourds, étonnés, gens sans faculté de jugement, pour lesquels être et ne pas être sont considérés comme la même chose et non comme la même chose. Leur chemin ramène au point de départ" (fr. 6.4-9). Et le fr. 7 résume cette description: "Et que l'habitude invétérée ne t'oblige pas, le long de ce chemin, à utiliser l'œil qui ne voit pas, l'ouïe qui ne renvoie qu'un écho, et la langue. Juge avec le lógos la réfutation très polémique que je viens d'énoncer" (fr.7.3-6).

Lorsque la Déesse, au fragment 1, avait présenté à l'apprenti philosophe son "programme d'études", elle l'avait incité à être au courant (puthéstai) non seulement du cœur imperturbable de la vérité mais aussi des "opinions des mortels", même si elles ne possédaient pas une "vraie conviction" (pístis alêthés) (fr. 1.30). Or, dans la suite du

\footnotetext{
${ }^{10}$ Noêsai, "pour penser" es infinitif aoriste actif. La plupart des traducteurs supposent que Parménide a écrit "noêttai" et traduisent "pensables", "concevables": "can be conceived" (Tarán, L. [1965], p. 32), "which are alone conceivable" (Coxon, A. H. [1986], p. 52), "les seules que l'on puisse concevoir / the only ones that can be thought of" (O'Brien, D.-Frère, J. [1987, II, p. 16).

${ }^{11}$ Une conjecture proposée par $\mathrm{H}$. Diels pour remplir une lacune au vers 6.3 faisait dire à la Déesse qu'il fallait s' "écarter" de cette première voie. Nous avons rejeté cette conjecture et proposé le verbe "commencer par". Cf. Cordero, N.L. (1979).

${ }^{12}$ Cette formule reprend, presque mot à mot, le vers $3 \mathrm{du}$ fr. 2 . Contre toute logique, la plupart des travaux consacrés à Parménide voient dans cette formule une allusion au "deuxième chemin", celui interdit par la Déesse. O'Brien, D. (1987, II, p. 25) par exemple, dit que dans "cette première voie de recherche [il y a] la mention du non-être". Il y a plus qu'une "mention": la Déesse dit que le non-être (repris par "néant") n'existe pas.

${ }^{13}$ Parménide lui-même fait allusion à sa méthode au fr. 5: "Il est commun pour moi où je commence, car j'y reviendrai à nouveau".
} 
Poème, les dóxai seront opposées au "raisonnement convaincant" (pistòn lógon) qui finit au vers $50 \mathrm{du}$ fr. 8 . La description impitoyable des mortels que nous venons de voir dans les dix vers consacrés à décrire leur état d'esprit justifie leur incapacité de raisonner (ils sont une ákrita phûla, fr. 6.7). La pístis est absente de l'ensemble des mots (kósmos...epéón, fr. 8.52) qu'ils utilisent pour décrire leurs dóxai, et, pour cette raison, il est "trompeur" (apatelón); ergo, il est l'opposé du "chemin de la persuasion" (peithoûs kéléuthos)" (fr. 2.4), qui est accompagné par la vérité. Nous reviendrons infra sur cette opposition "persuasion/tromperie".

Dans la suite de notre travail nous n'approfondirons pas la notion de pistón lógon que la Déesse expose jusqu'au vers $50 \mathrm{du}$ fr. 8. Il suffit de dire que celui-ci a clairement un point de départ ("Il ne reste qu'un chemin, celui d'éstin; sur ce chemin il a plusieurs preuves...", fr. 8.1-2) et un point final ("Je termine ici...", fr. 8.50), précisés par Parménide lui-même. ${ }^{14}$ Regardons, en revanche, ce que l'on peut dire sur les "opinions des mortels", notamment les citations récupérées qui développent la notion de "dóxa". Pour traiter ce dossier il faut mener à bien une recherche, car nous ne comptons pas, comme dans le cas du pistón lógon, d'un ensemble de cinquante vers (au fr. 8) qui s'occupe de la question. Nous avons une seule certitude: le début de l'exposé des dóxai ("À partir d'ici apprends les opinions des mortels....", 8.51), mais, à la différence du pistòn lógon, il n'y a pas un point d'arrivée. Jusqu'où va l'exposé des dóxai ?

Dans l'arrangement orthodoxe actuel des fragments, le fr. 19 semble exprimer la conclusion de l'explication de l'état actuel "des choses" selon la dóxa: "Ainsi son nées ces choses (éphu táde), selon l'opinion (katà dóxan), et elles sont présentes maintenant". Mais, où se trouvait cette citation, dans la version originale du Poème? Le hasard ${ }^{15} \mathrm{a}$ voulu qu'elle se trouve, aujourd'hui, à la fin des dix-neuf citations récupérées, ce qui a invité les chercheurs modernes à croire que toutes les citations précédentes (qui vont du fr. 9 jusqu'au fr. 18) faisaient partie de "La Doxa". Ceci est tout à fait arbitraire, car dans l'ensemble fr.9-fr.18 il y a, de toute évidence -on reviendra sur ce sujet- des fragments qui n'ont rien à voir avec ce que Parménide appelle "dóxaî". Quand nous avons dit que les

\footnotetext{
${ }^{14}$ Cependant, les auteurs partisans de trouver deux "parties" dans le Poème, "La Vérité et "La Doxa", placent aussi, dans l'ensemble "La Vérité", les fr. 2 à 7, ce qui est absurde, car dans les fr. 6 et 7 il y a des références au faux chemin, celui de la "dóxa".

${ }^{15}$ Elle a été placé à la fin du Poème tout simplement parce qu'elle a été découverte tardivement, en 1810, lorsque le texte avait été déjà rangé par Fülleborn (en 1795) en dix-huit citations. Si on l'avait découvert avant, l'actuel "fr. 19" aurait pu être placée dans un autre endroit.
} 
partisans de trouver des "parties" dans le Poème on dû choisir les citations qu'ils placeraient dans le dossier la dóxa, ils semblent ne pas avoir fait une recherche pour savoir qu'est-ce que cela veut dire, "dóxa", pour Parménide. Ils ont adopté, peut-être, inconsciemment, le préjugé aristotélicien de considérer que toute affirmation sur tà phainómena (par exemple, sur le soleil, la lune, les sexes, la structure du noûs) rentrait eo ipso dans le dossier "La Doxa". C'est le point de vue de Simplicius, concrétisé dans notre formule "la platonisation de Parménide": "Parménide place (títhesi) les corps parmi les choses 'opinables' (en tô̂s doxastô̂s)" (Phys., 87.5); "Parménide appelle 'opinable' (doxastón) ce qui est sensible (tò aísthêton)" (Phys., 38.26).

Nous avons dit que la description parménidienne des dóxai a un point de départ, le vers 8.51 , mais, déjà au beau milieu du fr. 8 (texte placé, par les partisans de trouver des "parties" dans le Poème, dans la "partie" "La Vérité" !), Parménide avait résumé l'activité des "mortels": "Ils ne sont que des noms ce que les mortels (brotoí) ont établi (kathétento), persuadés que c'étaient vrais" (fr. 8.38-9). Cette soudaine présence des "mortels" au fr. 8 (ainsi comme la description de leur statut aux fragments 6 et 7) relativise (et même annule) la prétendue division du Poème en deux "parties".

Revenons maintenant a la présentation détaillé des dóxai, à partir du vers 8.51: "À partir d'ici, apprends les opinions des mortels [...]. Ils ${ }^{16}$ ont établi deux points de vue (gnómas $)^{17}$ pour donner des noms à des formes extérieures [...]: d'une part, le feu éthéré de la flamme; [...] d'autre part, ce qui est en soi son contraire, la sombre nuit" (8.5359)...et la citation se termine deux vers après. Cependant, après cette citation du fr. 8 , le citateur, Simplicius, ajoute "...et peu après..." (kaí met'olíga pálin) (Phys. 180.8), ce qui suggère que la description résumée au fr. 8 pourrait continuer de cette manière: "...Mais comme tout a été nommé lumière et nuit [...] tout est plein en même temps de lumière et de nuit obscure, égales l'une à l'autre, car dehors d'elles il n'y a rien" (fr. 9). Et le couple lumière-nuit réapparaît aussi au fr. 12, où, même si le mot "dóxai" est absent, le diákosmon que le texte décrit correspond évidemment aux arkhaí des "mortels", le feu ou la lumière, et la nuit. Disons finalement que, mis à part les quatre textes que nous venons de citer (fr. 8.38-39, fr. 8.53-59, fr. 9 et fr. 12) aucun des fragments qui ont été mis arbitrairement dans la "partie" "La Doxa" ne correspond aux deux arkhaí, en dehors desquels, disent les "mortels" eux-mêmes, "il n'y a rien" (fr. 9.4).

\footnotetext{
${ }^{16}$ Peut-on affirmer, comme le feront les Doxographes, que Parménide parle de lui-même?

${ }^{17}$ Gnóme est synonyme de dóxa.
} 
Avant de continuer notre démarche, il faut faire une mise au point nécessaire, que nous n'avons pas la possibilité d'approfondir dans ce travail mais qui va conditionner la suite de notre recherche. Nous verrons infra que la doxographie parménidienne a trouvé chez l'Éléate, à côté du pistón lógon, des réflexions sur la "réalité sensible". Or, jusqu'à a la Sophistique - c'est notre avis - la dichotomie sensible-intelligible, ergo, apparencesêtre, est introuvable chez les premiers philosophes. L'application de la dichotomie aux Atomistes, par exemple, conduirait a un paradoxe qui n'aurait pas échappé à Zénon (s'il avait connu au moins Leucippe): les "principes" de tout ce qui est sensible sont l'atome et le vide, qui sont...intelligibles. C'est la Sophistique qui, pour justifier le relativisme (commun à tous les sophistes, sauf Antiphon), s'est appuyée sur les sensations, qui ne perçoivent pas "l'être" des choses, mais ce qui apparaît. Les sophistes ont établi cette dichotomie pour nier l'un des membres, l"l'être". En revanche, l'eón de Parménide, comme les arkhaí de ses prédécesseurs, est en dehors de la dichotomie être-paraître, d'où la difficulté des Doxographes, notamment ceux d'origine néoplatonicien, de comprendre des images "physiques", comme celle de la sphère, qui cohabitent avec le théorie de l'identité d'être et penser (fr. 3). Parménide n'est pas coupable de ce péché d'anachronisme.

Mais comme, grâce à Platon, qui a assumé la dichotomie sophistique et a pu revendiquer le sensible parce qu'il a trouvé une garantie de son existence, les Formes, les commentateurs des Présocratiques -notamment Aristote et son école, via Théophraste, et les Néoplatoniciens après- ont platonisé les premiers philosophes et leur ont demandé des réponses à des questions qui ont été posées des siècles après. C'est le cas de Parménide.

Comme, à partir de Platon, chaque philosophe s'est vu obligé de justifier la cohabitation du sensible avec l'intelligible (c'est à dire, des apparences avec "l'être"), déjà les interprètes anciens ont transféré rétroactivement le problème à Parménide (et nous verrons qu'Aristote dit que Parménide a été "obligé" de le faire). Regardons le cas de deux auteurs qui justifient notre affirmation. K. Bormann ${ }^{18}$, dans un article sur Parménide et Simplicius, a écrit: "The doctrines of Parmenides of the one being and the world of seeming were -as is well known-interpreted in different ways in the course of the history of philosophy", et B. M. Perrry, dans un travail sur le même sujet, n'hésite pas à commencer son livre par ces mots: "One of the central theses in Plato is the being-seeming

\footnotetext{
${ }^{18}$ Borman, K. (1979, p. 30).
} 
antithesis [...]. The obvious precursor for the antithesis, at any rate, is Parmenides, who rigidly distinguishes being from seeming or opinion in his Poem". ${ }^{19}$

Sans prétendre jouer le rôle d'avocat du diable, essayons de justifier cet anachronisme. C'est l'assimilation de tout ce qui est "physique" au sensible (soumis à la génération et la corruption, au mouvement, etc.), comme on voit clairement chez Platon, qui a invité Aristote (on verra après le cas de Platon) a exiger de Parménide un traitement aussi des réalités différentes de l'Être-Un. Platon, en revanche, n'a pas platonisé Parménide; il l'a tout simplement "mélissisé". ${ }^{20}$ Tel que Platon l'interprète, Parménide, qui, comme Mélissos, était un moniste -pour lui-, aurait nié l'existence d'un phaínesthai et d'un dokeîn (cf. la définition du sophiste comme un illusionniste, Soph. 236c), car, pour justifier l'existence de ces notions, il faudrait démontrer, contre Parménide, qu'il y a du non-être.

Voyons maintenant le cas d'Aristote. La plupart des références à Parménide se trouvent au Livre I de la Physique. Au début du chapitre 2 il est question des "principes" (arkhaî) et Aristote dit que ceux-ci, pour les phusikoí, sont en mouvement, tandis que pour Parménide (et Mélissos) le principe est un et immobile. Mais dans d'autres passages aussi bien de la Métaphysique que de la Physique, Aristote dit que Parménide, en ce qui concerne la multiplicité sensible, avait aussi proposé, comme tous les phusikoú, deux principes contradictoires. ${ }^{21}$ Voici comment Aristote justifie l'existence d'un Parménide phusikós et ou phusikós à la fois: "Tous [les phusikoí] posent des contraires comme principes [....] Même Parménide pose le chaud et le froid comme principes, et il les nomme feu et terre" (Phus. 188a19-21). Parménide donc, n'est pas une exception. Un grand penseur comme lui ne pouvait pas nier l'existence de la multiplicité sensible (car "examiner l'eón un et immobile n'est pas examiner la phúsis", Aristote, Phus. 184b27). Et c'est ainsi comme "Parménide, obligé de suivre les phénomènes (anagkazómenos d' akoloutheîn toîs phainómenois), et, tout en ayant affirmé que, selon le lógos, [l'être] est

\footnotetext{
${ }^{19}$ Perry, B.M. (1983, p. 11).

${ }^{20}$ Voir l'interprétation littérale de Platon des vers 8.43-45 de Parménide (qui, en réalité, fait une analogie entre l'homogénéité du fait d'être et la masse d'une sphère) selon laquelle il conclut que "l'être a un milieu et extrémités" (Platon, Sophiste 244e). Cette prétendue "spatialité de l'être est propre de Mélissos, qui a écrit que, étant donné que le vide n'existe pas, l'être est Un, car il occupe tout l'espace (paraphrase du fr. 30 B 7 DK).

${ }^{21}$ Malgré lui, et mutatis mutandis, Parménide, grâce à Aristote, semble devancer de plusieurs siècles la très connue phrase de Groucho Marx: "Voici mes principes; si vous ne les aimez pas, je vous en donne d'autres".
} 
un, même si, selon la sensation, il est multiple, supposa deux causes et deux arkhaí, le chaud et le froid, c'est-à-dire, le feu et la terre; il rangea l'un, le chaud, comme être, et l'autre comme non-être" (Met. 986b31) ${ }^{22}$. Le raisonnement d'Aristote est impeccable, mais s'il avait lu la totalité du Poème $e^{23}$ il aurait compris que tout ce qu'il dit est attribué par Parménide... aux "mortels qui ne savent rien".

C'est vrai que chez Parménide il y a des références à des gens qui ont posé comme principes (dans le sens aristotélicien du mot) le chaud et le froid, ou le feu ou la lumière et la terre (il suffit de lire les vers 8.55-59), mais, comme nous avons vu, Parménide ne partage pas cette conception. C'est Aristote, le premier, qui a affirmé, avec force et dans plusieurs endroits de ses travaux, la paternité parménidienne de ce "point de vue" (gnóme $)$ pour expliquer la réalité: "Parménide a fait du chaud et du froid des arkhaî" (Phys. 188a19); "il a placé deux causes ou principes, le chaud et le froid" (Mét. 986b34); "ceux qui ont placé deux éléments, comme Parménide, le feu et la terre" (De Gen. 330b13), etc. Théophraste s'est évidemment inspiré de son maître (nous reviendrons sur son témoignage) dans son Phusikôn doxônn, source, a son tour de l'ensemble de la doxographie parménidienne, jusqu'à Simplicius (à l'exception, apparemment, de Sextus Empiricus et de Plutarque $\left.{ }^{24}\right)$.

Or, un phénomène très curieux s'est produit entre l'écriture du Poème de la part de Parménide, et Simplicius, son dernier citateur: il s'agit de la disparition totale de la description très sévère des "mortels" o "les hommes" en tant que créateurs des opinions et, par conséquence, de leur incompétence pour expliquer tà phainómena. Même les

\footnotetext{
${ }^{22}$ Ce passage avait déjà étonné Ross, W. D. (1924, I, p. 134).

${ }^{23}$ Il est probable que dans l'Antiquité il y avait déjà, à côté du texte complet du Poème (que Simplicius avoue posséder encore dans son temps, onze siècles après son écriture), des résumés soit de ses idées les plus originales, soit des parties qu'on avait bien comprises, car, dans certains domaines, il semblait partager les recherches des philosophes précédents. L'article fondamental de Mansfeld, J. (1986) démontre l'existence, même avant Platon, d'ouvrages de classification des philosophes anciens, soit des notions générales, soit des principes. Il est très curieux que le texte connu aujourd'hui comme "fragment 2", d'où sort la vulgata "L'Être est et ne non-Être n'est pas", soit demeuré inconnu pendant onze siècles (!), jusqu'à sa citation de la part de Proclus et Simplicius. Avant notre ère (jusqu'à Plutarque) seulement quelques vers de quatre fragments de Parménide ont été cités: fr. 7 (Platon, Aristote), fr. 8 (Platon, Eudème), fr.13 (Platon, Aristote) et fr.16 (Aristote, Théophraste).

${ }^{24}$ Cf. Perry, B. M. (1983, p. 65). Selon Palmer, Plutarque s'inspirerait directement d'Aristote (2009, p.39).). Mais dans Adv. Col. 1115 Plutarque dit que Colotès ne pouvait pas ignorer les travaux de Théophraste. On peut donc supposer que lui non plus. Cependant, il aurait pu connaître Théophraste sans pour autant s'inspirer de lui.
} 
Doxographes qui ne se prononcent pas d'une manière claire et distincte sur la paternité parménidienne de la doctrine (Parménide aurait exposé ce que l' "on dit"), évitent toute référence aux critiques que Parménide lui-même adresse aux "mortels". C'est le cas de Théophraste qui, plus prudent que son maître, aurait dit (apud Alexandre, Dox. 482) que

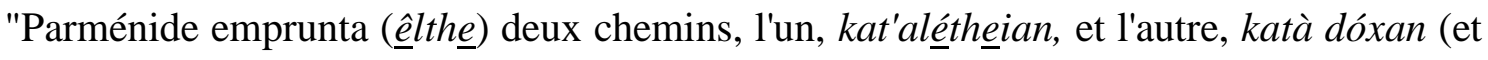
celui-ci suppose comme arkhaí le feu et la terre)". Mais Théophraste aurait pu critiquer la contradiction qui suppose l'assimilation de la terre ou du froid au non-être, qui, selon Parménide, n'existe pas. Alexandre, de son côté, suppose que, dans le cas de tà phainómena Parménide aurait suivi l'opinio communis (celle de tôn pollôn) (apud Simplicius, Phys. 38). Hippolyte, enfin, dans un autre contexte, dit que Parménide ne s'écarte pas de la dóxa pollôn en ce qui concerne les principes (Ref. I.2 = DK A 23). Dans tous les autres cas, de Plutarque à Simplicius, on affirme sans hésitation que c'est Parménide lui-même qui a expliqué le doxastón (= le sensible) par l'action de deux principes, le feu (principe actif) et la terre (principe passif). Dans tous les cas, le commentaire critique de Parménide au dualisme des mortels ("Ils n'ont pas fait une unité des formes extérieures, en quoi ils se sont trompés", fr. 8.54) est totalement ignoré.

Pour mener à bien cette entreprise de désinformation (volontaire? conséquence de la lecture d'une copie défectueuse ou mutilé du texte de Parménide?) il fallait surmonter un inconvénient: la référence très précise de Parménide aux auteurs qui ont proposé l'explication dualiste du doxastón, les "mortels" ou, en général, "les hommes", et à la critique parménidienne qui s'en suivait. Or, d'Aristote à Simplicius, aucun Doxographe n'a fait référence au sujet. Ils semblent s'incliner face à la solution la plus facile: si Parménide a fait une place aux dóxai dans son Poème, elles reproduisent sa pensée, comme dit Palmer. ${ }^{25}$ Le poids du dualisme platonicien, renforcé par les nombreuses références chez Aristote au caractère opposé des arkhaí que l'on trouve chez les Présocratiques, a eu comme résultat une interprétation de la philosophie de Parménide dans laquelle on a privilégié ces deux notions (qui se trouvent réellement dans le Poème), et on a laissé de côté le caractère "trompeur" (apatelón) de la théorie doxique. Comme nous hésitons à admettre un Parménide díkranos, qui aurait eu deux manières différentes de penser, nous respectons le style du discours de la Déesse: quand elle parle à la première personne, elle est le porte-parole de Parménide; quand elle utilise le "ils", elle parle

\footnotetext{
${ }^{25}$ Palmer (2009, p. 32).
} 
d'autres auteurs, même si notre post-modernité (et peut être déjà celle des Doxographes, par rapport à l'époque de Parménide) nous empêche de les individualiser.

Au début de ce travail nous avons fait allusion au poids qui a chaque mot dans un texte qui est arrivé jusqu'à nous d'une manière lacunaire. Les nombreuses références à ces deux mots, "mortels" et "hommes" (huit fois; voir infra) ainsi que la longue (dix vers) description de la "condition humaine", méritent d'attirer l'attention de n'importe quel chercheur.

Nous avons dit au début de ce travail que l'état plus que lacunaire dont le Poème a été récupéré, nous oblige à étudier chaque mot comme s'il était un joyau. Dans le cas de Parménide, la plupart des trois cents quatre-vingt (environ) mots conservés, ne sont utilisés qu'entre une et quatre fois. Il y a certainement, deux exceptions: le vocabulaire concernant le verbe "être" (eînai, eimí, eón, etc.), ainsi que les mots qui sont en rapport avec la notion de "pensée" (noeîn, noémata, etc.), qui sont un développement de l'énigmatique fr. 3, "être et penser sont la même chose". Dans ces deux cas, la terminologie est très abondante. Et, comme le Poème va s'occuper de la manière dont la vérité et les "opinions des mortels" envisagent le fait d'être (eón), les mots concernant ces deux sujets dépassent aussi la moyenne habituelle que l'on déduit de l'utilisation des autres mots. C'est ainsi comme on trouve 3 fois alétheia $(1.29,2.4,8.51)$ et 4 fois ale thés $(1.30$, $8.17 ; 8.28,8.39)$ et -c'est notre sujet- 4 fois ánthropoi $(1.27,16.2,16.3,19.3)$ et 4 fois brotoí ("mortels", qui résume la "condition humaine" (1.30, 6.4, 8.39, 8.61). Certainement, ces mots auraient dû être beaucoup plus abondants dans la version complète du texte, mais si nous considérons que les citations récupérées (appelées des "fragments") sont en réalité des "morceaux choisis", parce qu'elles contiennent des affirmations ou des thèses significatives, non anodines, il faut essayer de nous expliquer la surabondance d'utilisations que nous avons constatée des mots "mortels" et "hommes" chez Parménide, ainsi que les dix vers consacrés à décrire le status de ceux-ci, ignorés par la totalité des Doxographes.

Nous sommes très sceptiques sur ce qu'on appelle le "passage du múthos au lógos" 26 , mais "comme tous les êtres humains désirent naturellement savoir" (Aristote, Mét. 980a1), lorsque les premiers philosophes (qui étaient considérés des "sages") ont mis en marche, chacun selon sa voie particulière, des "méthodes" pour "savoir" d'une manière la plus objective et impersonnelle possible, ils se sont trouvés face à des "opinions",

\footnotetext{
${ }^{26}$ Voir Cordero, N. L. (2017, pp. 21-23).
} 
parfois anonymes, parfois "personnalisées", qui les avaient précédés. La rareté des écrits authentiques des penseurs de Milet nous oblige à commencer le traitement de ce sujet ${ }^{27}$ par Xénophane. Ses critiques s'adressent surtout aux "opinions" à propos des (ou de "la") divinités. Selon Xénopnane, Homère et Hésiode "ont attribué aux dieux tout ce qui est objet de honte et de reproche" (fr. 11), et au fr. 14, il utilise le même mot que Parménide, "mortes": "Les mortels (brotoí) croient (dokéousi, verbe de la famille de dóxa) que les dieux sont nés". "Il n'y aura jamais un homme (anèr)" capable de posséder une connaissance précise (saphès) sur les dieux; il aura accès (tetuktai) seulement à une opinion" (dókos). Par conséquent, il faut opiner (dedoxástho) que "les choses qui semblent (eoikóta) [sont] réelles (etúmoisin)" (fr. 35). Pour Xénophane, donc, si nous écoutons "les hommes", nous sommes condamnés à rester dans le domaine des "opinions".

Dans le cas d'Héraclite, ses critiques des opinions "des autres", sont proverbiales. Sa victime préférée est Pythagore (fr. 81, fr. 129), ainsi comme la polumathía d'Hésiode, Xénophane et Hécatée (fr. 40). La dóxa, avec la signification de "ce que l'on dit", n'est pas crédible, elle peut tromper: "Le plus renommé (dokimótatos) connaît et protège des opinions" (dokéonta)" (fr. 28). Mais l'ennemi principal d'Héraclite est la foule: "La plupart (polloî) ne comprend pas les choses telles qu'ils les rencontrent, ni même après les avoir rencontré; elles leur 'paraissent' (dokéousi) (fr. 17); "Les hommes (ánthropoi) se laissent tromper dans la connaissance (gnôssin) des choses évidentes" (fr. 56); "Les opinions (doxásamata) sont [comme] des jouets d'enfants" (fr. 70); "[Les hommes] se laissent persuader par les chanteurs populaires (démon) et la foule (homíloi) est leur maître; ils ne comprennent pas que le grand nombre (hoi polloî) sont méchants (kakoî), et [seulement] quelques-uns sont bons" (fr. 104). C'est dans ce contexte qu'il faut interpréter les références de Parménide aux "mortels qui ne savent rien", sur lesquelles les Doxographes n'ont rien dit.

Même une lecture superficielle du Poème montre qu'il s'appuie sur des images ou notions opposées (des "dichotomies"), dont l'un des membres il faut retenir et l'autre doit être abandonné. Au fr. 1 il y a les chemins de la nuit et du jour, et "l'homme qui sait" doit être au courant du cœur de la vérité et des opinions; au fr. 2 la Déesse expose deux manières d'acheminer une recherche (bien ou mal); au fr. 6 et 7 on décrit le chemin qui affirme le fait d'être (ésti gàr ềnai) et rejette l'existence du néant (6.1-2); au fr. 7 un

\footnotetext{
${ }^{27}$ Voir Cordero, N. L. (2019, pp. 47-51).
} 
chemin et abandonné et le premier vers du fr. 8 dit que, en conséquence, un chemin a été laissé de côté et on a retenu celui qui est "authentique" (8.16-7). Et avant (8.11) il avait dit qu' "il faut exister absolument ou ne pas exister du tout". Dans tous les cas, comme les "mortels" croient qu'être et ne pas être sont la même chose et non la même chose, ils sont incapables de "juger" (ils sont ákritoi, et le vers 8.16 a présenté une krísis) et choisissent (mal) la conjonction (lumière et nuit; dans la doxographie, chaud et froid). Parménide dit que, dans ce cas, "ils se sont trompés" (8.54). Pour respecter le schéma général du Poème, toute cohabitation de la vérité, qui aurait comme objet d'étude "L'Être", et les dóxai, qui s'occuperaient des "apparences" est inimaginable. Mais depuis Aristote et jusqu'à Simplicius les Doxographes ont dit le contraire, et les historiens de la philosophie l'on répété, jusqu'à aujourd'hui.

Nous arrivons au point central de notre travail. Revenons en arrière. Nous avons déjà dit que la dichotomie sensible-intelligible était inconnue avant la Sophistique. Parménide n'a pas besoin de justifier "le sensible", catégorie qui, pour lui, n'existe pas (le réel n'a pas besoin de cette catégorie pour exister: une "chose" est "ce qui est", tò eón, un "étant", grâce auquel "l'être" "est présent", car eón est le participe présent du verbe "être"), et, évidemment, il n'a pas été obligé de le faire, comme suppose Aristote (Mét. 986b). Mais ceci ne veut pas dire qu'il ne s'est pas intéressé à des réalités appelées après "physiques" ou "sensibles". Le fr. 10, placé d'une manière totalement arbitraire dans "La Doxa" par ceux qui divisent le Poème en deux "parties", est clair et précis. La Déesse, à la première personne, exhorte l'apprenti philosophe à "apprendre (eísei) la phúsis" des astres, ainsi que "la phúsis de la lune tournante" et à "apprendre (eidéseis)" qu'est-ce que le ciel, etc. Quand un Présocratique (et peut être déjà Homère: cf. $O d$. X.302) utilise le mot phúsis il fait référence à l'être de quelque chose (Homère, d'un phármakon). Ces objets, qui seront après appelés "sensibles" (lune, astres, ciel, sexes), sont susceptibles d'un "savoir" (verbe eidénai) qui concerne leur phúsis, donc, leur être. Héraclite, presque en même temps que Parménide, écrira que la sagesse consiste à expliquer "chaque chose selon la phúsis" (fr. 1). Une fois que nous savons qu'il y a de l'être (et que cet "état" est caractérisé par toute une série de sémata), ce "principe", qui est "le cœur de la vérité", explique tout, car il est "convaincant".

Or, depuis Platon, lorsque le philosophe est obligé de rendre compte d'une réalité dédoublée (sensible + intelligible), on demande aux penseurs préplatoniciens de s'adapter à cette nouveauté. Le paradigme de cette position est Aristote: "il est évident qu'eux aussi énoncent certains principes et certaines causes" (Mét. 983b3). Même si les mots 
"principe" et "cause" ne se trouvent pas chez Parménide, il y a chez lui l'exposé d'une diakósmesis qui s'appuie sur des "éléments", grosso modo, le feu et la terre, mais qui n'ont pas joué le rôle qu'Aristote assigne aux "principes"; ils ont été utilisés pour "nommer" toutes choses (cf. fr. 9). Et, surtout, ils n'ont pas cohabité avec une explication du réel non-sensible (?). En plus, Parménide oppose ces opinions au "cœur de la vérité", car il ne les partage pas. Une fois que la Déesse a appris au jeune voyageur qu'est-ce que la vérité et que sont les opinions "des mortels", un choix s'impose, car les deux activités proposent une explication du même sujet: la réalité ${ }^{28}$. Le fr. 7 confirme qu'un chemin a été abandonné et qu'il ne reste qu'une voie, celle d' "on est" (ésti, 8.1); l'hypothèse des "opinions" a été abandonnée (fr. 7.3). Bref, une éventuelle thèse parménidienne sur le "sensible" (doxastón) est une invention des Doxographes, et ceci, déjà à partir d'Aristote.

$\mathrm{Si}$, dans chaque cas, on avait ajouté que la Déesse dit d'une manière claire et précise que les opinions sont un produit des "autres", et qu'elle se limite à les exposer pour les critiquer, on aurait respecté ce que nous trouvons dans les citations récupérées (et que la cohérence qui caractérise la méthode parménidienne n'aurait pas pu contredire dans des passages non conservés). Il ne s'agit pas, chez les Doxographes, d'une mauvaise interprétation, mais tout simplement d'une faute, qui est évident même dans le citateur le plus talentueux et important, Simplicius. En effet, après avoir écrit qu' "il [= Parménide] établit (étheto), lui aussi, comme principes [...] la lumière et l'obscurité, ou le feu et la terre", il ajoute cette citation littérale de Parménide: "Ils ont établi (katéthento) deux points de vue [...]; d'une part, le feu éthéré [...]; d'autre part, ce qui est en soi son contraire, la sombre nuit" (8.53-9; Simpl. Phus. 30). Comment n'a t-il pas remarqué qu'il attribue à "il" et à "ils" la même théorie?

Si nous avons eu la désinvolture d'utiliser la formule "le très curieux silence" pour qualifier la disparition des références toujours négatives aux créateurs de l'explication dualiste du doxastón que décrit (sans la partager) la Déesse de Parménide, c'est parce que nous ne trouvons pas une réponse acceptable pour offrir au lecteur éventuel de ces pages. Nous avons vu supra que, depuis Aristote, on a essayé à tout prix de trouver chez les Présocratiques au moins des allusions aux premiers principes et au premières causes, mais il ne suffit pas de trouver des termes qui font allusion aux éléments (comme le feu et la terre dans le cas de Parménide); il faut évidemment comprendre l'utilisation que fait

${ }^{28}$ Cf. Palmer, J. (2009, p. 42): "Theophrastus understood Parmenides' 'Truth' and 'Doxa' as accounts of the same thing first in its intelligible and then in its phenomenal aspects". 
l'auteur de cette terminologie. Platon, dans le Théétète, commente et même cite un texte original de Protagoras, mais cela ne fait pas de Platon un partisan du relativisme sophistique. En réalité, tout a commencé par Aristote ${ }^{29}$ et son soupçon de la nécessité qui aurait poussé Parménide à devenir un phusikós comme les autres. Peut-être par politesse, d'abord Théophraste et après les Doxographes néoplatoniciens, se sont interdits de parler de manière négative des "mortels", ce qui aurait déstabilisé le Maître, mais ceci serait inexplicable dans le cas où l'on cite le Poème, comme Simplicius.

Nous avons dit supra qu'on n'a pas le droit de demander à Parménide des réponses à des questions qui ne faisaient pas partie de la panoplie des "sages" avant le tsunami de la Sophistique, comme la justification d'un éventuel univers sensible (que Platon appelait tò doxastón; cf. la ligne divisée de la République). Pour trouver une réponse à ce sujet on a forcé le texte parménidien à dire ce qu'il ne voulait pas dire, on a supprimé toutes les références négatives aux "mortels", et on a groupé les citations du Poème en "parties", bref, on a commis un véritable parménidicide.

En revanche, il est tout à fait légitime de vouloir trouver chez Parménide, comme chez ses collègues, une "physique" (= une étude de la phúsis) ou des essais d'explication de tà ónta, car "il n'y pas des ónta qui ne soient pas" (fr. 7.1). La Déesse, à la première personne, incite le jeune voyageur à "connaître" la phúsis des astres, etc. (fr. 10), et le fr. 16 propose une explication "physique" de la structure du noûs. Mais Parménide n'admet pas une dakósmessis qui s'appuie sur des arkhaí contradictoires et que, au lieu de chercher une vérité, croit déjà la posséder et, par conséquent, l'impose (il fait une utilisation très abondante du verbe katatíthemi) à la réalité. En revanche, il encourage des recherches qui s'appuient sur ce que, dans sa philosophie, est un véritable axiome (et non un arkhé): il y a de l'être (eón émmenai, fr. 6.1). ${ }^{30}$

L'alternative parménidienne n'est pas "sensible/intelligible", et même pas "vrai/faux", mais "convaincant/trompeur", étant donné le caractère didactique de son Poème (cf. Note 3). La voie de recherche à suivre est convaincante (c'est la voie de peithoûs); le chemin des "mortels" est trompeur (apatelón ${ }^{31}$ ). Or, toute explication de la

\footnotetext{
${ }^{29}$ Cf. Cordero, N. L. (2016).

${ }^{30}$ Cf. Cordero, N.L. (2011, pp. 95-113).

${ }^{31}$ Conditionné par son l'interprétation platonico-aristotélicienne de Parménide, le mot apatelón dérange Simplicius, qui essaie de le nuancer: "'Trompeur' ne veut pas dire 'absolument faux' (pseudê haplôss), mais comme tombé (? ekpeptokóta) de la vérité intelligible sur les phénomènes
} 
phúsis qui suppose qu'il y a de l'être (avec les sémata énumérés au fr. 8), et qui est convaincante, aurait dû se trouver dans la version originale du Poème, avant le vers 8.50, qui clôt le pistón lógon. C'est le cas de la plupart des fragments mis arbitrairement dans la "partie "La Doxa" (fr. 10, 11, 13, 14, 15 $5^{32}$, 16, 17, 18) ainsi comme des textes, aujourd'hui inconnus, dans lesquels se trouveraient certaines thèses vraies et convaincantes mentionnés par les Doxographes, par exemple que la terre est sphérique, qu'elle se trouve au centre de l'univers, que l'âme et l'intellect sont la même chose, que les astres sont des boules de feu, que le soleil s'est séparé de la voie lactée, que la terre peut se diviser en cinq zones, que dans la vieillesse il y a une augmentation du froid, etc. ${ }^{33}$

Comme dans un mauvais roman policier, ce travail a détecté un délit (une disparition), a détecté les auteurs du délit (Aristote et les Doxographes, jusqu'à Simplicius), a détecté les complices du délit (les experts modernes qui l'ont occulté), mais a été incapable de trouver à qui profite le délit. Quoi qu'il en soit, il est évident qu'il ne profite pas à Parménide, car, étant donné l'absence du coupable, on lui a attribué la paternité d'une théorie qu'il a exposé, mais pour la critiquer.

Nestor-Luis Cordero

(Université de Rennes 1, Professeur Emérite)

sensibles" (Phys. 39). Il semble ne pas saisir que Parménide n'utilise jamais le mot pseudê (au moins dans les citations récupérées), car "trompeur" est l'antithèse de "vrai".

${ }^{32}$ Ce n'est pas vrai et convaincant que la lune est toujours tournée vers les rayons du soleil?

${ }^{33}$ Dans les dernières années, Rossetti, L. a mené à bien des recherches très approfondies sur ces sujets (2017). Cf. un commentaire admiratif mais cependant critique sur sa position in Cordero, N.L. (2017). 


\section{Bibliographie}

Bormann, K. "The interpretation of Parmenides by the neoplatonist Simplicius", The Monist, 62, 1979.

Cordero, N. L. "Las 'partes' del Poema de Parménides: un prejuicio interpretativo trágico", Mathémata, Ecos de Filosofía Antigua, ed. R.Gutiérrez, Lima: Fondo Editorial de la Pontificia Universidad Católica de Perú, 2013

Cordero, N. L. "La Déesse de Parménide, maîtresse de philosophie", La naissance de la raison en Grèce, ed. J.-F. Mattéi, Paris: PUF, 1990.

Cordero, N. L. "Parmenidean 'Physics' is not a part of what Parmenides calls 'dóxa", in Parmenides, venerable and awesone, ed. N.L.Cordero, Las Vegas/Zurich/Athens Parmenides Publishing, 2011, 95-113.

Cordero, N. L. By Being, It Is, Las Vegas: Parmenides Publishing, 2004.

Cordero, N. L. La invención de la filosofía, Buenos Aires : Biblos, 2017.

Cordero, N. L. Les deux chemins de Parménide, Paris/Bruxelles: Vrin/Ousia, $1997^{2}$.

Cordero, N. L., "Aristote, créateur du Parménide díkranos que nous héritons aujourd'hui", Anais de filosofia clássica, X, 19, 2016.

Cordero, N. L., "Il y a, évidemment, un «Parménide phusikós», mais...(A propos de Rossetti, L. Un altro Parmenide, 2017)", Archai, 25, 2019.

Cordero, N. L., "Les deux chemins de Parménide dans les fragments 6 et 7", Phronesis, 24, 1979.

Cordero, N. L., Heráclito: uno es todo, todo es uno, Buenos Aires : Colihue, 2018.

Coxon, A. H. The fragments of Parmenides, Assen: Van Gorcum 1986.

Galgano, N. S. "Graduação e pós-graduação em Parmênides DK 7", Educación, arte y política en la filosofía antigua, V. Suñol y M. Berrón [eds.], Asociación Argentina de Filosofía Antigua, Libro digital PDF, 2020.

Jaeger, W. The theology of the early greek Philosophers, Oxford: Oxford University Press, 1947.

Mansfeld, J. Aristotle, Plato and the Preplatonic Doxography and Chronography in Storiografia e dossografia nella filosofia antica, ed. G. Cambiano, Torino, 1986.

O'Brien, D. ; Frère, J. Études sur Parménide, ed. P. Aubenque, Paris: Vrin, 1987

Palmer, J. Parmenides and presocratic philosophy, Oxford, 2009.

Perry, B. M. Simplicius as a source for and interpreter of Parmenides, Thèse, University of Michigan 1983.

Ross, W. D. Aristotle's Metaphysics, Oxford 1924, vol. 1.

Rossetti, L. Un altro Parmenide, 2 volumes, Bologne : Diogene, 2017.

Tarán, L. Parmenides, Princeton: Princeton University Press, 1965. 